\title{
Alltagsleben und politische Partizipation - Gecekondu-Viertel als gesellschaftlicher Ort
}

Everyday life and political participation - Gecekondu district as a social place

\section{Heidi Wedel}

\section{OpenEdition}

\section{Journals}

Electronic version

URL: https://journals.openedition.org/ejts/140

DOI: $10.4000 /$ ejts. 140

ISSN: $1773-0546$

Publisher

EJTS

Electronic reference

Heidi Wedel, „Alltagsleben und politische Partizipation - Gecekondu-Viertel als gesellschaftlicher Ort " European Journal of Turkish Studies [Online], 1 | 2004, Online erschienen am: 04 März 2015, abgerufen am 26 Mai 2021. URL: http://journals.openedition.org/ejts/140 ; DOI: https://doi.org/10.4000/ejts.140 
Citation: Wedel, Heidi (2004) 'Alltagsleben und politische Partizipation - Gecekondu-Viertel als gesellschaftlicher Ort', European Journal of Turkish Studies, Thematic Issue N 1 - Gecekondu, URL: http://www.ejts.org/document140.html To quote a passage, use paragraph (§).

\title{
Altagsleben und politische Partizipation - Gecekondu-Viertel als gesellschaftlicher Ort
}

Heidi Wedel

\begin{abstract}
This article demonstrates how gecekondus as a specific place prestructure social space and political participation. Gendered place and gendered space in gecekondus lead to gendered political priorities and action. Housing security is a "felt need" shared by all squatters. Yet regarding infrastructure and social institutions, men focus on the wider city and demand better connection to urban traffic and improved institutions on the district level. On the other hand, women whose social and work life is widely restricted to the neighborhood, are primarily concerned about deficiencies of infrastructure within the neighborhood. Whereas men may assume formal roles in politics, women are widely excluded from the male-dominated public sphere and related resources of political participation. Their political action is rather based on spatial and social resources of participation such as informal networks, which are facilitated by place and space in gecekondus. Women's political participation is mainly of an informal nature. The article discusses the effects and constraints of their collective actions regarding political awareness, local leaders and formal political participation.
\end{abstract}


Citation : Wedel, Heidi (2004) 'Alltagsleben und politische Partizipation - Gecekondu-Viertel als gesellschaftlicher Ort', European Journal of Turkish Studies, Thematic Issue $N^{\circ} 1$ - Gecekondu, URL http://www.ejts.org/document140.html

To quote a passage, use paragraph (§).

Gecekonduviertel sind Wohnviertel, die zwar in Städten oder am Rande von innen liegen, sich aber physisch und soziologisch von anderen Stadtvierteln unterscheiden. Ein überwiegender Teil ihrer Bewohnerinnen sind Stadtmigrantinnen, die entweder direkt nach der Übersiedlung vom Dorf in die Stadt in ein Gecekondu zogen oder nach einer Zwischenstation in einer Mietswohnung1. Gecekondus sind Häuser, die - wie im Gecekondugesetz von 1966 definiert - ungeachtet von Grundbesitzverhältnissen und/oder Baugenehmigungen und regulierungen gebaut werden. Bei der Gründung sind oft keinerlei Infrastruktur und soziale Einrichtungen vorhanden. In neu entstehenden Vierteln sind die Häuser zunächst sehr weitständig gebaut, u.a. um die anschließende Ansiedlung von Verwandten und hemşehris, Menschen aus demselben Dorf oder Kreis, in der nächsten Nähe zu gewährleisten. Sie ähneln dann nach Kıray Vierteln der unteren Mittelschicht oder - was treffender ist - durchschnittlichen kleinen Dörfern. Durch ständigen Zuzug, der auch der materiellen und physischen Absicherung dient, wird die Siedlungsdichte immer größer, und die Infrastrukturprobleme werden brennender (Kıray 1972 : 564). Es handelt sich also um Stadtviertel, die sowohl physisch als auch soziologisch gesehen gewisse dörfliche Merkmale aufweisen.

[2] Von Wissenschaftlerinnen werden oft drei Phasen des Gecekondubaus unterschieden, so z.B. bei Keleş (1983 : 211 und 1993 : 381 f): (1) bis 1960: "unschuldig", von den Bewohnern selbst mit geringer Hilfe gebaute Häuser zur Befriedigung der Wohnbedürfnisse von armen Familien ; (2) 1960-70: mit Eigenarbeit der Bewohner gebaut, aber nicht nur für den Familienbedarf, sondern darüber hinaus zum Vermieten ; (3) 1970-80: vollständige Kommerzialisierung des Baus von Häusern zum Verkauf an arme Familien, keinerlei Selbstarbeit mehr, überwiegend von Mietern bewohnt. Auch Köksal (1990 : 260 f.) unterscheidet die "klassischen" Gecekondus der frühen Phase schneller Urbansierung als nicht kommerzialisiert, vollständig außerhalb des Marktes, gebaut aus Altmaterialien mit reinem Gebrauchswert von den späteren, legalisierten und kommerzialisierten Gecekondus, deren Materialien auf dem Markt gekauft wurden und bei denen Besetzer, Erbauer

1 Nach Karpat (1976: 239, n. 3) sind schätzungsweise $90 \%$ der Gecekondubewohnerinnen Binnenmigrantinnen. Auf Seite 2 zitiert er allerdings eine nicht weiter qualifizierte Regierungsstudie, nach der $84 \%$ der Gecekondus von Migrantinnen bewohnt sind. Nach Tatlıdils Erhebungen in Kayseri waren nur 3,5\% der Haushaltsvorstände, die zwischen 1950 und 1960 die ersten Gecekondus gebaut hatten, in Kayseri geboren, unter den Bewohnern 1984-86 waren es $9 \%$. Daß der Anstieg so niedrig ist, zeigt in Anbetracht des Generationenwechsels, daß ein Großteil der Erbauer bzw. ihrer Nachkommen das Viertel verlassen hat (Tatıdil $1989: 57$ ). 
Citation : Wedel, Heidi (2004) 'Alltagsleben und politische Partizipation - Gecekondu-Viertel als gesellschaftlicher Ort', European Journal of Turkish Studies, Thematic Issue No1 - Gecekondu, URL : http://www.ejts.org/document140.html

To quote a passage, use paragraph (§).

und Bewohner nicht mehr identisch sind². Die starre Zuordnung von verschiedenen Gecekondutypen zu bestimmten Phasen ist jedoch insofern irreführend, als auch in den späteren Phasen ärmlichere Gecekondus weitgehend von den Bewohnern selbst gebaut wurden und insofern verschiedene Gecekondutypen gleichzeitig nebeneinander bestanden.

[3] In diesem Artikel wird untersucht, wie der spezifische physische Raum in Gecekonduvierteln eine soziale Sphäre vorstrukturiert, die nicht nur das Alltagsleben, sondern auch die politische Partizipation der Bewohnerinnen beeinflußt und in welcher Form die Bewohnerinnen zu lokalen Problemen aktiv werden. Ein besonderes Augenmerk gilt den stärker betroffenen Frauen, die aufgrund geschlechtsspezifischer Konzepte und Arbeitsteilung seltener als Männer das Gecekonduviertel verlassen und deren Arbeitsleben sich auch meist im Gecekonduviertel abspielt, während die meisten Männer außerhalb des Viertels arbeiten. Unter Rückgriff auf Barbara Holland-Cunz unterscheide ich zwischen materiellem Raum und sozialer Sphäre, "die gemeinsam die gesellschaftliche Praxis der in ihnen agierenden Frauen und Männer [stiften]. Räume schaffen oder verhindern Kommunikation, erleichtern oder erschweren Interaktion, gerade auch für Frauen. Aus sozialwissenschaftlicher Perspektive kommt dem gestaltenden Raum aber nur das Potential zu, gesellschaftliche Sphäre zu strukturieren und vorzustrukturieren. Der materielle Raum stellt nicht aus sich selbst heraus etwa Öffentlichkeit oder Privatheit her; gesellschaftliche Praxis muß inn erst durch Atmosphäre, Diskurs, Handeln etc. mit einem spezifischen Sinn und Bedeutungsgehalt füllen." (Holland-Cunz 1992/93 : 36) Im folgenden werde ich diskutieren, welche geschlechtsspezifischen Räume und Sphären (gendered place and gendered space) sich in Gecekonduvierteln bilden und wie dies auf die geschlechtsspezisch unterschiedlichen Möglichkeiten und Formen der Politikpartizipation wirkt.

[4] Der Artikel beruht auf Feldforschung in den Jahren 1992 bis 1997 mit einem Schwerpunkt in der Zeit vor und nach den Kommunalwahlen von 1994. Während dieser Feldforschung arbeitete ich mit einer bewußten Mischung qualitativer Methoden (teilnehmende Beobachtung, Einzel- und Gruppeninterviews sowie Experteninterviews) in unterschiedlichen

21996 bezeichnet Köksal, nun Erder, nur die ärmlicheren Behausungen als Gecekondus. Insbesondere seit der gewaltsamen Eskalation von städtischen Konflikten 1996 wird der mit Legitimität konnotierte Begriff Gecekondu durch varoş (eigentlich: "Viertel am Stadtrand") ersetzt, der auch das Bedrohungsgefühl der städtischen Elite zum Ausdruck bringt. 
Citation : Wedel, Heidi (2004) 'Alltagsleben und politische Partizipation - Gecekondu-Viertel als gesellschaftlicher Ort', European Journal of Turkish Studies, Thematic Issue No1 - Gecekondu, URL : http://www.ejts.org/document140.html

To quote a passage, use paragraph (§).

Gecekonduvierteln in Istanbul. Das Hauptuntersuchungsgebiet, das Viertel A, liegt im Bezirk Beykoz ${ }^{3}$.

\section{Charakteristika des Untersuchungsgebietes}

[5] Der Stadtteil A ist eines der Gecekondugebiete, die auf den Beykozer Hügeln in einem Gebiet, das früher der Waldbehörde (Orman Genel Müdürlüğü) unterstellt war, entstand. Berichten jetziger Bewohnerinnen zufolge wurde das Gebiet Ende der 40er/Anfang der 50er Jahre von drei Leuten aus der Schwarzmeerregion aufgeteilt und verkauft. 1976 lebten in A nach Aussagen des Muhtars İlyas nur 5-10 Familien aus der Schwarzmeerregion. Danach begann die intensive Besiedlung des Gebietes. Weil Rodung und Abbrennen des Waldes anhielten, verlor das Gebiet allmählich den Charakter eines Waldes.

[6] In der zweiten Hälfte der 70er Jahre begannen neben den Schwarzmeerleuten auch alevitische Bewohnerinnen aus Malatya nach A zu ziehen und bildeten dort ihr eigenes Quartier, das aus 3 Gassen besteht und das "kurdische Viertel" genannt wird4. Auch die anderen Zuwanderinnen siedelten sich in der Nähe ihrer Verwandten und hemşehris an, so daß im älteren Kern von A verschiedene Quartiere auszumachen sind, die ethnisch, religiös und bezüglich der Herkunft relativ homogen sind: Neben dem schon erwähnten "kurdischen Viertel" sind dies die Quartiere der Leute aus Kastamonu, Giresun und Ordu. Dieses sind auch die größten regionalen Gruppen im Viertel. Die amtierenden Muhtare der 90er Jahre stellen die hemşehri-Gruppen unter den Wahlberechtigten im Jahr 1994 in folgender Spannbreite dar: 33-41 $\%$ der Wählerinnen aus Kastamonu, 12-19 \% aus Giresun, 11-14 \% aus Ordu und 7\% Bewohnerinnen aus Malatya. Am Rande des Quartiers der Leute aus Ordu lebt auch eine kleine Gruppe alevitischer Bewohnerinnen aus Adana. Diese Quartierbildung im Zuge der Kettenmigration ist seit Ende der 80er Jahre nicht mehr möglich, weil sich nach 1983 die Besiedlung nochmals beschleunigte und der Boden so knapp wurde, daß Neuzuziehende bauen mußten, wo sie noch eine Lücke fanden.

3 Zu den Details der Untersuchung und ihren Ergebnissen, siehe Wedel (1999).

$4 \mathrm{Im}$ Unterschied zum "Stadtteil" als kleinster Verwaltungseinheit, vertreten durch einen Muhtar, verwende ich den stadtplanerischen Fachbegriff "Quartier" für den sozial in sich geschlossenen Siedlungsraum ethnischer oder religiöser communities innerhalb eines Stadtteils (Dubetsky 1977 : 361). Im Türkischen werden dagegen beide als mahalle bezeichnet, was von mir auch mit dem Begriff "Viertel" wiedergegeben wird. 
Citation : Wedel, Heidi (2004) 'Alltagsleben und politische Partizipation - Gecekondu-Viertel als gesellschaftlicher Ort', European Journal of Turkish Studies, Thematic Issue $N^{\circ} 1$ - Gecekondu, URL http://www.ejts.org/document140.html

To quote a passage, use paragraph (§).

[7] 1983 wurde A, das damals ca. 1.000 Einwohner hatte, offiziell zu einem Stadtteil. Seitdem ist die Einwohnerzahl sprunghaft angestiegen, betrug bei der Volkszählung 1990 knapp 6.000, im Oktober 1993 nach Angaben des damaligen Muhtars Illyas 10-12.000. Dieser Anstieg liegt v.a. an dem Baugesetz von 1985, den Bauamnestien von 1984 und 1985 und den anschließenden neuen Bebauungsplänen: ab 1986 wurde der Stadtteil infrastrukturell für die Bebauung erschlossen und der Kernbereich, der mittlerweile von der Bezirksverwaltung gekauft worden war, parzelliert; Grundbucheintragungen wurden ermöglicht. Daneben spielt der Bau der zweiten Bosporusbrücke eine herausragende Rolle : Bewohnerinnen aus Nachbarvierteln, deren Gecekondus wegen des Brückenbaus 1985 abgerissen wurden, kauften sich mit der Entschädigung ein Gecekondu in A, und die Grundstückspreise stiegen aufgrund der verbesserten Verkehrsanbindung, was zu einer Gentrifizierung führte.

[8] Im Viertel gibt es ein kleines Zentrum mit einer Moschee und Läden. Von der Hauptstraße gehen Gassen ab, die z.T. sehr steil nach unten laufen. Auch einzelne kleine Grundstücke weisen eine extreme Hanglage auf. Da ohne technische Hilfe gebaut wird, kommt es immer wieder zu Abrutschungen. Mit der Vergrößerung des Viertels wird in immer ungünstigeren Lagen gebaut, selbst in den Bachtälern, was besonders gesundheitsgefährdend ist. Hier leben tendenziell ärmere Leute. In A wohnen die Familien noch überwiegend in ihren eigenen Gecekondus, Mieter gibt es kaum. In den 90er Jahren hatte A weitgehend den Charakter eines Gecekonduviertels mit kleinen, einstöckigen bzw. durch die extreme Hanglage zweistöckigen, leicht gebauten Häusern, die weiß oder farbig getüncht sind. Sie sind von Gärten umgeben, in denen Obstbäume stehen und z.T. auch Gemüse angepflanzt wird, so daß insbesondere die älteren Teile von A grün und dörflich wirken. In den 90er Jahren sah man nur vereinzelt aus Stahlbeton gebaute, zwei- bis dreistöckige moderne Häuser, die oft mit Unterstützung von in Deutschland lebenden Verwandten gebaut wurden. An der Hauptstraße zeichnete sich aber schon ein rapider Wandel ab. Hier wurden moderne, z.T. sehr repräsentativ gebaute Wohnhäuser mit Gewerberäumen im Erdgeschoß gebaut, während die gegenüberliegende Seite, auf der 1993/94 noch vereinzelte Gecekondus standen, von einer großen Baufirma erworben wurde, die hier teure Apartmenthäuser errichtet und internationale Holdings ansiedelt. Es ist zu vermuten, daß die derzeit überwiegend armen Bewohnerinnen von A durch diesen Wandel allmählich verdrängt werden bzw. ihre bisherige räumlich extensive Wohnkultur mit regen sozialen Kontakten innerhalb der Quartiere verändern müssen. 
Citation : Wedel, Heidi (2004) 'Alltagsleben und politische Partizipation - Gecekondu-Viertel als gesellschaftlicher Ort', European Journal of Turkish Studies, Thematic Issue $N^{\circ} 1$ - Gecekondu, URL http://www.ejts.org/document140.html

To quote a passage, use paragraph (§).

[9] Schon 1977 wurde die Grundschule eröffnet, 1994 eine Mittelschule. Die Moschee wurde ab 1988 mit Spenden, die über einen Moscheeverein gesammelt wurden, gebaut. In ihrem Untergeschoß gibt es Läden, die vermietet werden. Daneben ist auch eine Apotheke. Eine Gesundheitsstation wurde erst nach der Kommunalwahl 1994 eingerichtet. Einen Wochenmarkt gibt es in A nicht.

[10] Zu Beginn der Besiedlung 1975 gab es keinerlei Infrastruktur in A. Da es weder Busse noch Straßen gab, mußten Berufstätige und Schülerinnen mit einem langen, beschwerlichen Fußweg vom Bosporus aufsteigen. Jetzt fahren Stadtbusse nach Üsküdar, ins Zentrum von Beykoz und nach Mecidiyeköy, was v.a. für Bewohnerinnen, die im Verwaltungsund Dienstleistungssektor arbeiten, von Bedeutung ist. Die Haltestelle ist aber so abgelegen, daß die Benutzung für Frauen nach Einbruch der Dunkelheit unangenehm ist. Eine kompliziertere und teurere Verbindung nach Topkapı ist v.a. für alle interessant, die im Kleingewerbe arbeiten. Die Gassen innerhalb des Viertels wurden erst im Winter 1992/93 bzw. vor der Wahlwiederholung im Juli 1994 betoniert, oft jedoch nur die oberen Stücke, so daß die Bewohnerinnen der unteren Gassenabschnitte im Winter weiterhin durch den Schlamm waten müssen. Strom- und Telefonleitungen wurden in den 80er Jahren gelegt, Kanalisation jedoch noch nicht in allen Teilen von $A$.

[11] Ein großes, erst im September 1997 gelöstes Problem ist die Versorgung mit fließendem Wasser. Die Frauen bohrten Wasserleitungen an, um sich selbst illegal mit Wasser zu versorgen, oder mußten das Wasser aus verschiedenen Quellen selbst heranholen. Den ersten Wasseranschluß erhielten die Gecekondus an der Hauptstraße 1978/79, die Gassen wurden über Brunnen versorgt und erst - wenn überhaupt - Mitte der 80er Jahre an das städtische Wassernetz angeschlossen. Aber auch hier kam 1993/94 das Wasser sehr unregelmäßig und hatte zu wenig Druck für die höher gelegenen Gassen. Im September 1994 ließ die RP-geführte Bezirksregierung Gecekondus abreißen, um dort 1997 eine Pumpstation in Betrieb zu nehmen. Zwischenzeitlich wurde, wo es keine Wasserversorgung gab, je nach politischer Phase und Druck der Bewohnerinnen Wasser mit Tanklastwagen von der Bezirksregierung geliefert, jedoch nie ausreichend. Alternativ waren die Frauen auf Brunnen- und Quellwasser oder teures, privat geliefertes Wasser angewiesen. 
Citation : Wedel, Heidi (2004) 'Alltagsleben und politische Partizipation - Gecekondu-Viertel als gesellschaftlicher Ort', European Journal of Turkish Studies, Thematic Issue N¹ - Gecekondu, URL : http://www.ejts.org/document140.html

To quote a passage, use paragraph (§).

\section{Soziale Situation der Bewohnerinnen}

[12] Nach einer allgemeinen Bewertung der sozialen Situation von A gefragt, bezeichnen die Muhtare der 90er Jahre die Bewohnerinnen als arm. Nach dem früheren Muhtar İlyas sind Dreiviertel arbeitslos oder ohne Kranken- und Rentenversicherung beschäftigt, nach dem neuen Muhtar Mustafa sind $50 \%$ arbeitslos und die meisten unversichert beschäftigt. Es gebe 30-100 Kleingewerbetreibende (esnaf), 50 Ladenbesitzer, 100 Beamte, 200 Rentner und 500 Straßenhändler. Als im Viertel angesiedeltes Gewerbe zählen sie 11 (Illyas) bzw. 5 (Mustafa) Teehäuser, 3 Garküchen (Iokanta), je ca. 30 Gemischtwarenläden (bakkal) und Obst- und Gemüsehändler (manav), zwei Tischler und eine Autowerkstatt auf. Daneben gibt es noch einen Brotbäcker und gegenüber dem Friedhof einen Marmorbearbeiter.

[13] Das monetäre Einkommen der Familien ist schwer zu berechnen, da es sich aus mehreren, oft unregelmäßig fließenden Quellen zusammensetzt. Der Grad der sozialen Absicherung kann besser beurteilt werden, wenn an Fallbeispielen die Lebensbedingungen und die jeweilige Kombination verschiedener Überlebensstrategien verdeutlicht werden. Die folgenden Beispiele zeigen, daß ein regelmäßiges Arbeitseinkommen zwar eine Form der minimalen Grundsicherung bietet, im Arbeitsmarkt jedoch kaum Aufstiegschancen gegeben sind bzw. dieser durch Unsicherheit geprägt ist. Deshalb müssen die Erwerbsaktivitäten durch andere Formen der langfristigen Absicherung ergänzt werden, welche sich v.a. auf den Immobiliensektor beziehen und/oder durch die Pflege von informellen Netzwerken erreicht werden.

[14] Die 40jährige Hayriye zog als Verwandte eines Viertelgründers schon 1974, als es in A nur vereinzelte Familien gab, hierher. Sie kam im Alter von 11 Jahre mit ihren Eltern aus einem Dorf in Giresun nach Istanbul. Sie durfte nach dem Grundschulabschluß keine weiterführende Schule besuchen und wurde mit 17 Jahren verheiratet. Sowohl ihre jüngere Schwester als auch ihre eigenen fünf Kinder erlebten dagegen durch die Migration einen enormen Bildungsaufstieg: Die Schwester studierte und eröffnete ein eigenes Anwaltsbüro. Hayriyes Tochter Gamze war eins der ersten sunnitischen Mädchen aus $A$, die das Gymnasium abschlossen, und studiert mittlerweile Kunst.

[15] Ökonomisch hat die Familie überdurchschnittliche Möglichkeiten: Der Mann hat als öffentlicher Angestellter ein regelmäßiges Einkommen und eine Sozialversicherung sowie so gute Beziehungen, daß die Familie schon Ende der 70er Jahre einen Wasseranschluß bekam. Hayriye verkaufte früher die Milch inrer Haustiere. Später 
Citation : Wedel, Heidi (2004) 'Alltagsleben und politische Partizipation - Gecekondu-Viertel als gesellschaftlicher Ort', European Journal of Turkish Studies, Thematic Issue No1 - Gecekondu, URL : http://www.ejts.org/document140.html

To quote a passage, use paragraph (§).

machte sie Heimarbeit und ließ andere Frauen arbeiten, bis ihr Arbeitsvermittler zahlungsunfähig wurde. Durch diese Einkünfte sowie den Verkauf von Goldarmreifen - die Form, in der Frauen Kleinvermögen bekommen und aufbewahren - konnten sie das Gecekondu erwerben und später die Anzahlungen für eine Grundbucheintragung beginnen. Als die Raten 1994 abbezahlt waren, verfügte die Familie über einträgliches Immobilienvermögen, denn sie "besaß" ein wertvolles $900 \mathrm{~m}^{2}$ großes Grundstück direkt an der Hauptstraße. Da dieses bei weitem die maximale Größe für Gecekonduparzellen überschritt, wurde es in drei Parzellen eingeteilt, eine gehört Hayriye, eine ihrem Neffen und eine unbebaute der Bezirkskommune. Die Familie lebt weiterhin in dem Gecekonduhaus auf Hayriyes Parzelle, hat aber auf der zweiten Parzelle einen pompösen, mehrstöckigen Neubau gebaut, in dessen Keller der Neffe einen Billardsalon betreibt. Der Laden im Erdgeschoß ist noch nicht vermietet. Mit dem Neubau hat sich die Familie zunächst völlig übernommen, sobald sie wieder Geld hat, will sie über dem Laden eine Wohnung ausbauen.

[16] Insofern ist diese Familie ein Beispiel für Bildungsaufstieg über drei Generationen sowie einen ökonomischen Aufstieg, der ermöglicht wurde durch ein sicheres Einkommen des Mannes sowie die verwandtschaftlichen Beziehungen der Frau zu einem der "Viertelgründer" ergänzt durch den Einsatz ihrer Arbeitskraft und ihres Kleinvermögens. Diese Hauptbedingungen, Beziehungen und regelmäßiges Einkommen, fehlen Ayşe im Quartier der Leute aus Kastamonu :

[17] Ayşes Gecekondu ist sehr ärmlich : Im Vorraum, der als Küche dient, ist nackter Steinboden. Die sechsköpfige Familie schläft in dem sehr kleinen Wohnzimmer. Ayşe muß das Wasser aus einem Brunnen holen, weil das Haus keinen Wasseranschluß hat. Ihr Mann hat sich nicht darum gekümmert, weil er vermutet, daß das Haus wegen der Straßenplanung abgerissen wird.

[18] Ayşe (Mitte 40) hat ihren Mann und drei erwachsene Söhne sowie ihre bettlägerige Mutter zu versorgen. Die Männer tragen weder ausreichend zum Haushaltseinkommen bei noch helfen sie bei der Hausarbeit, sondern behindern sie, indem sie lange schlafen und dadurch das Wohnzimmer blockieren. Da Ayşe nicht mehr bereit war, die vier Männer zu versorgen und dabei noch nicht einmal ausreichend Geld zu haben, versorgt sie den eigenen Haushalt aus Protest nur noch oberflächlich und nahm eine Putzstelle an, um Geld zu verdienen.

[19] Da ihre Männer der Pflicht, für das Haushaltseinkommen zu sorgen, nicht nachkommen, verweigert sie ihre Aufgaben innerhalb der geschlechtsspezifischen Arbeitsteilung 
Citation : Wedel, Heidi (2004) 'Alltagsleben und politische Partizipation - Gecekondu-Viertel als gesellschaftlicher Ort', European Journal of Turkish Studies, Thematic Issue $N^{\circ} 1$ - Gecekondu, URL : http://www.ejts.org/document140.html

To quote a passage, use paragraph (§).

und stellt ihren begrenzten Wohnraum für regelmäßige Koranlesungen einer Gruppe sunnitischer Frauen zur Verfügung, ohne auf männliche Fürsorgeansprüche Rücksicht zu nehmen. So ist Ayşe trotz der ärmlichen Verhältnisse nicht sozial isoliert ${ }^{5}$. Die Familie von Gülistan steht mitten in einem noch unentschiedenen Kampf um ein würdiges, abgesichertes Leben:

[20] Gülistan kam mit 16 Jahren mit ihren Eltern und Geschwistern aus einem Dorf in Malatya nach Istanbul, wo der ältere Bruder das Gymnasium besuchte, während die Schwestern in einer Teppichwerkstatt arbeiteten. Im Alter von 20 Jahren heiratete sie Haydar, der in A im Quartier der alevitischen Kurdinnen aus Adana lebte, wo er mit sehr viel Eigenarbeit ein Gecekondu gebaut hatte. Das Haus wurde am Hang gebaut und hat eine kleine Souterrainwohnung, die gegen geringe Einnahmen vermietet wird. Die eigentliche Wohnung hat ein Wohnzimmer und zwei Schlafzimmer. Die Aufnahme einer unverheirateten Schwester von Haydar wird von Gülistan angesichts der eingeschränkten räumlichen und finanziellen Verhältnissen der Familie als zusätzliche Belastung empfunden. Die junge Frau hilft jedoch Gülistan bei Kinderbetreuung und Hausarbeit.

[21] Haydar hatte bis zum kommunalen Machtwechsel 1994 eine feste Stelle als Arbeiter bei der Kommune, erhielt jedoch wegen Budgetproblemen monatelang keinen Lohn. Als seine Partei die Regierung und er deshalb die Arbeit verlor, arbeitete er als Reinigungskraft in einem Hotel auf der europäischen Seite. Der Mindestlohn, den er dort erhielt, reichte nur in Verbindung mit der Abfindung, die er von der Kommunalverwaltung erhalten hatte. Als Haydar noch ein regelmäßiges Gehalt bezog, zahlte er in eine Wohnungsbaukooperative ein, von der er sich eine langfristige ökonomische Absicherung erhoffte.

[22] Gülistan nutzt jede freie Zeit, die neben der Hausarbeit und der Versorgung der fünf- bzw. sechsköpfigen Familie bleibt, um in Heimarbeit Kleinartikel herzustellen. Außerdem hilft sie in einem Laden in A aus, der von Haydars jüngerem Bruder betrieben wird und in dem ein Teil des Einkommens der Großfamilie erwirtschaftet wird. Das von einem weiteren Bruder, der in Deutschland lebt, überwiesene Geld ermöglichte Investitionen wie die in den Laden sowie den Kauf eines Grundstückes im "kurdischen Viertel". Dort wird ein fünfstöckiges Haus für Haydars Eltern und Geschwister bebaut, und zwar mit sehr viel Eigenarbeit von Haydar und seinen Nachbarn und Verwandten in Etappen. Haydar, Gülistan und ihre Kinder bleiben aber weiterhin in ihrem Gecekondu wohnen. 
Citation : Wedel, Heidi (2004) 'Alltagsleben und politische Partizipation - Gecekondu-Viertel als gesellschaftlicher Ort', European Journal of Turkish Studies, Thematic Issue $N^{\circ} 1$ - Gecekondu, URL http://www.ejts.org/document140.html

To quote a passage, use paragraph (§).

[23] Nur durch eine Kombination verschiedener Einkommensquellen und Strategien, v.a. aber durch die Unterstützung innerhalb der Großfamilie, Immobilieninvestitionen und die Mobilisierung sozialer und politischer Kontakte kann sich diese Familie ohne regelmäßiges Einkommen vor einem Abrutschen in die Armut schützen. Während die dafür eingesetzte Arbeitskraft des Mannes durch Erwerbstätigkeit und Hausbau offen sichtbar ist, erfolgt der Beitrag der Ehefrau über Heimarbeit und Mitarbeit im Laden fast unmerklich "nebenbei". Außerdem leistet sie über die Mitversorgung von Mitgliedern der Großfamilie ihren Anteil zur Pflege dieses Netzwerkes, das der Familie den Hauptschutz gewährt. Nicht zuletzt ist sie über eine aktive Mitgliedschaft in der Partei, die damals die Kommunalregierung stellte, auch an dem Aufbau und Erhalt der politischen Kontakte beteiligte, welche wiederum die Voraussetzung sowohl für Haydars feste Stelle als auch für den Erwerb des Grundstückes und die Erteilung einer Baugenehmigung für den Neubau waren.

\section{III „Empfundener Bedarf“ als Voraussetzung für politische Aktion}

[24] Wie oben dargestellt ist der Stadtteil weiterhin von typischen Problemen der Gecekonduviertel geprägt, weil die Bebauung noch weitgehend illegal ist und Infrastruktur sowie soziale Einrichtungen noch größere Mängel aufweisen bzw. ganz fehlen. Bei zunehmender sozialer Differenzierung unter den Bewohnerinnen gehören viele von ihnen immer noch zu den ärmeren städtischen Schichten und verfügen über keine längerfristige soziale Absicherung. Der weiterhin bestehende Bedarf an städtischen Dienstleistungen könnte also zur Entstehung von politischen Aktivitäten im Stadtteil führen. Ob es zur Bildung von Stadtteilorganisationen kommt, hängt davon ab, welchen Bedarf die Bewohnerinnen subjektiv wahrnehmen, ob ihnen Ressourcen für politische Partizipation zur Verfügung stehen und ob eine kollektive Identität kollektives Handeln ermöglicht.

[25] Welchen Bedarf Gecekondubewohnerinnen "empfinden", habe ich in den drei Blöcken Wohnsicherheit, Infrastruktur und soziale Einrichtungen untersucht (Wedel 1999 : 127-144). Meine geschlechtsbewußte Analyse zeigt, daß nur Wohnsicherheit ein von allen Bewohnerinnen angestrebtes Ziel ist, während die Bedürfnisse an Infrastruktur und sozialen Einrichtungen je nach Geschlecht unterschiedlich gewichtet werden. Dies hängt einerseits mit der geschlechtsspezifischen Arbeitsteilung, nämlich der weltweiten Zuständigkeit von Frauen für reproduktive Aufgaben zusammen. Andererseits sind Gecekondufrauen sehr viel stärker als Männer von den Bedingungen 
Citation : Wedel, Heidi (2004) 'Alltagsleben und politische Partizipation - Gecekondu-Viertel als gesellschaftlicher Ort', European Journal of Turkish Studies, Thematic Issue $N^{\circ} 1$ - Gecekondu, URL http://www.ejts.org/document140.html

To quote a passage, use paragraph (§).

im Stadtteil betroffen, weil sie weitergehender als z.B. lateinamerikanische Squatterfrauen aus der männlich dominierten Öffentlichkeit ausgeschlossen und räumlich auf den Stadtteil eingeschränkt sind.

[26] Weil Gecekondus eine - wenn auch prekäre - Wohnsicherheit bieten und das knappe Familienbudget von Mietausgaben entlasteten, sind sie ein Pfeiler der sozialen Absicherung. Sie können aber langfristig auch zur Grundlage eines Vermögens in Form von Immobilienbesitz werden, bieten also Möglichkeiten der vertikalen sozialen Mobilität. Frauen leiten wie Männer aus der von ihnen in das Gecekondu gesteckten Arbeitskraft einen Rechtsanspruch auf Haus und Boden ab: „Wir Frauen haben diese Grundstücke durch unsere Arbeitskraft (emek) erworben“. Sie stellen selbstbewußte Forderungen an den Staat, der ihre illegale Ansiedlung tolerierte. Dabei fordern sie die Vergabe von Bodentiteln (tapu). Umstritten sind die tapu-Preise, Größe und Lage. Nach Angaben des Muhtars İlyas konnte nur ein Viertel der Bewohnerinnen 1986 Bodentitel erwerben. Die restlichen Bewohnerinnen müssen heute viel höhere Preise bezahlen. Für die meisten Bewohnerinnen von $A$ ist also langfristige Wohnsicherheit (noch) nicht greifbar. Für sie sind die Preise eines tapu ein zentrales Anliegen in ihrer Auseinandersetzung mit staatlichen Stellen und der Verhandlung mit politischen Parteien. Sie argumentieren entweder, daß die Preise ihre finanziellen Möglichkeiten überschreiten, was auf ein sehr niedriges, unregelmäßiges Einkommen schließen läßt, oder daß ihre Wohnsituation zu unsicher sei, weil ihr Gecekondu evtl. wegen öffentlicher Bauvorhaben oder des Verkaufs ihrer Grundstücke an zahlungskräftigere Personen oder Firmen vom Abriß bedroht sei, sich also eine finanzielle Investition nicht lohnen würde. Angenommene Abrißgefahr und die Höhe der tapu-Preise waren auch der Auslöser einer großen kollektiven Aktion, ein mehrtägiger Sitzstreik des ganzen Stadtteils vor dem Bezirksrathaus.

[27] Bei Familien, die eine Legalisierung ihres Bodens und Hauses finanzieren können, tritt das Streben nach größtmöglichem Gewinn aus Immobilien in den Vordergrund. Wenn die Kommunalverwaltung soziale Argumente wie die Versorgung möglichst vieler städtischer Armer mit Wohnraum aufgreift und bei der Legalisierung Höchstgrenzen für Gecekonduparzellen festlegt, fordern die Bewohnerinnen, daß die faktischen „Eigentumsverhältnisse“ vom Staat ohne Abstriche berücksichtigt werden.

[28] "Ich kann es nicht ertragen, wenn sie meinen Boden anderen geben wollen. Ich habe dort Bäume, einen Brunnen, Obst. Ich habe soviel Arbeit hineingesteckt, wie können sie das anderen geben? Ich 
Citation : Wedel, Heidi (2004) 'Alltagsleben und politische Partizipation - Gecekondu-Viertel als gesellschaftlicher Ort', European Journal of Turkish Studies, Thematic Issue No1 - Gecekondu, URL : http://www.ejts.org/document140.html

To quote a passage, use paragraph (§).

kann denen ja auch das tapu-Geld dafür geben. Warum bringen sie alles durcheinander? Wenn jemand sich direkt vor meiner Tür niederläßt, werden wir natürlich Probleme miteinander bekommen. Seit 22 Jahren bewahre ich das [Grundstück]. Ich schütze es nach allen Seiten hin. Wie kann das jemand weggeben?... Natürlich, es gehört dem Staat, aber wir haben inm ja auch dafür Geld gegeben. Wir haben seit Jahren einen Zuteilungsschein (tapu tahsis belgesi) für das ganze Grundstück, wir zahlen seit Jahren Steuern dafür. Warum war der Staat denn damals nicht dagegen?"

[29] Wegen des von ihnen empfundenen Rechtsanspruches sind sie auch bereit, das Gecekondu notfalls gewaltsam gegen staatliche Abrißversuche oder die Ansiedlung von anderen Migrantinnen zu verteidigen. Diese Gewalt sehen sie als soziale Notwehr und insofern legitim, als sie der Befriedigung ihres Bedürfnisses nach bezahlbarem Wohnraum dient. Die Gecekondubewohnerinnen verteidigen aber nicht nur ihre Wohnsicherheit und damit ein Grundbedürfnis, sondern - selbst wenn dies explizit verneint wird - auch den Erhalt ihrer raumgreifenden, durch dörfliche Gewohnheiten beeinflußten Wohnweise. Dieses Interesse wird jedoch nicht kollektiv vertreten, weil es auch mit Streben nach einer weitergehenden materiellen Absicherung der eigenen Familie durch möglichst großen Immobilienbesitz verbunden ist. Problematisch ist, daß dadurch die Ansiedlung für neue Migrantinnen immer schwieriger wird, was durch Bodenspekulation und Kostenanstieg noch verschärft wird. Schließlich wird die soziale Heterogenität innerhalb der Viertel noch weiter verstärkt. Selbst zu dem allen Squattern gemeinsamen Bedürfnis nach Wohnsicherheit haben also die Familien abhängig von ihrer ökonomischen Situation unterschiedliche Vorstellungen.

[30] Die Bedürfnisprioritäten bezüglich der Infrastruktur und sozialen Einrichtungen spiegeln zusätzlich die geschlechtsspezifische Arbeitsteilung wider: während die meisten Männer außerhalb des Stadtteils arbeiten und sich daher eher für öffentliche Verkehrsanbindung zum Arbeitsplatz und ggf. Kulturangebote und politische Bildungsmöglichkeiten auf der Bezirksebene interessieren, haben Frauen ihren Lebens - und Arbeitsschwerpunkt im Stadtteil. Sie knüpfen an ihren reproduktionsbezogenen Aufgaben an und fordern zu ihrer Arbeitserleichterung v.a. Wasserversorgung und Straßenbefestigung. Denn es sind die Frauen, die das Wasser herbeischleppen oder bei verschlammten Wegen ihre Schulkinder bis zur nächsten befestigten Straße tragen, ihnen Plastiktüten um die Schuhe binden und täglich Schuhe und Kleidung reinigen. Der Mangel an Infrastruktur, v.a. an Wasser, wird von allen Bewohnerinnnen, insbesondere aber 
Citation : Wedel, Heidi (2004) 'Alltagsleben und politische Partizipation - Gecekondu-Viertel als gesellschaftlicher Ort', European Journal of Turkish Studies, Thematic Issue $N^{\circ} 1$ - Gecekondu, URL http://www.ejts.org/document140.html

To quote a passage, use paragraph (§).

den Frauen, als dringender lokaler Bedarf gesehen. Akute Probleme werden oft durch informelle, teils auch illegale Selbsthilfe individuell oder manchmal kollektiv gelöst, wobei die Netzwerke der Nachbarinnen mobilisiert werden. Diese soziale Solidarität sollte jedoch nicht romantisiert werden, denn sie dient letzlich der Versorgung der einzelnen Familien und findet hier auch ihre Grenzen. Nur in seltenen Fällen reicht sie aus, um kollektive Aktionen durchzuführen oder mit Kosten und Arbeit verbundene gemeinsame formelle Lösungen zu verwirklichen.

[31] Sind die Grundbedingungen des Wohnens, eine feste Bleibe und eine Grundversorgung mit Infrastruktur erreicht, und haben sich die einzelnen Gecekondus zu einer Siedlung konstituiert, ist also ein gemeinsamer lokaler Raum entstanden, gewinnt eine weitere Kategorie an lokal angesiedelten Bedürfnissen an Bedeutung, nämlich die nach sozialen Einrichtungen im Viertel, mit denen wiederum Grundbedürfnisse zweiten Grades wie Bildung, Gesundheit, Kultur und Freizeitgestaltung befriedigt werden. Während einige Familen viel Geld investieren, um ihre Kinder auf bessere Schulen außerhalb des Stadtteils zu schicken, stellt für viele Familien schon der Besuch der örtlichen Grundschule eine ökonomische Belastung dar. Die Mütter kritisieren v.a. die Zwangsbeteiligung der Eltern an den laufenden Kosten der Schule, aber auch die unhygienischen Verhältnisse. Für Männer spielen diese Probleme bei der Befragung keine Rolle.

[32] Daß geschlechtsspezifisch unterschiedliche Bedürfnisprioritäten auch zu Spannungen führen können, zeigt das zweite wichtige Anliegen von Frauen in A, die Forderung nach Einrichtung eines Wochenmarktes in ihrem Stadtteil. Dies würde für die Frauen nicht nur weniger Zeitaufwand bedeuten, sondern innen auch helfen, das knappe Haushaltsbudget besser zu nutzen. Nach dem kommunalen Regierungswechsel treten sunnitische Frauen aus Kastamonu, hemşehris des neuen Muhtars, in Verhandlung. Sie vermuten, daß der seit zwei Jahren geplante Markt durch die Besitzer der Läden unter der Moschee verhindert wird, die den Wochenmarkt wegen seiner günstigeren Preise als Konkurrenz fürchten. Als die Frauen wiederholt gegenüber dem innen persönlich bekannten Beigeordneten des Muhtars auf die Einrichtung eines Marktes drängen, behauptet dieser, die Markthändler würden die Frauen und Mädchen des Viertels belästigen, und bringt sie schließlich mit der sexistischen Beleidigung "Wenn deine Tochter keinen Mann findet, willst du dann auf dem Markt einen Mann für sie suchen?" zum Schweigen. Er versucht also, die Frauen in ihrer sexuellen Identität zu treffen, um 
Citation : Wedel, Heidi (2004) 'Alltagsleben und politische Partizipation - Gecekondu-Viertel als gesellschaftlicher Ort', European Journal of Turkish Studies, Thematic Issue $N^{\circ} 1$ - Gecekondu, URL http://www.ejts.org/document140.html

To quote a passage, use paragraph (§).

zu verschleiern, daß eigentlich ökonomische Individualinteressen verteidigt werden. Vielleicht wollte er aber auch verhindern, daß mit dem Wochenmarkt ein neuer öffentlicher Ort für Frauen entsteht, an dem sich Frauen unterschiedlicher ethnischer Zugehörigkeit begegnen und an dem neben Waren auch Informationen ausgetauscht werden können. Denn in anderen Vierteln wird neben dem ökonomischen Aspekt auch die soziale Funktion des Wochenmarktes für Frauen mit geringem räumlich/sozialen Radius betont.

[33] Die bisher genannten Bedürfnisse erwachsen aus der gegebenen Arbeitsteilung und stellen diese nicht in Frage. Diesbezüglicher akuter Bedarf wird von den Frauen meist informell, teils auch illegal, individuell, durch soziale Selbsthilfe gelöst, in selteneren Fällen durch kollektives Vorsprechen bei kommunalen Stellen. Andere auf soziale Einrichtungen bezogene Forderungen der Frauen haben dagegen auch strategischen Charakter, indem sie auf neue öffentliche Räume und Zusammenhänge für Frauen (Märkte, Frauencafes) und eine Organisierung und Sichtbarmachung ihrer produktiven Tätigkeiten (Frauenwerkstätten, Organisierung der Heimarbeit) abzielen, also die Veränderung von Geschlechterverhältnissen anstreben. Männer nehmen diese Forderungen entweder nicht zur Kenntnis oder arbeiten aktiv gegen ihre Umsetzung.

\section{Ressourcen politischer Partizipation und kollektive Identität}

[34] Das Bewußtsein lokaler Probleme wird nur selten in kollektive Aktionen oder Organisation umgesetzt, weil insbesondere den vorrangig betroffenen Frauen die in den Sozialwissenschaften vorausgesetzten Ressourcen politischer Partizipation (McCarthy, Zald 1977) nur eingeschränkt zur Verfügung stehen. In meiner Arbeit habe ich die Wirkung des Mangels an Ressourcen wie formelle Bildung, bei Kurdinnen auch Sprachkenntnisse, Geld, Zeit, angesehene oder wenigstens außerhäusliche Stellung, Organisationserfahrung, räumliche Mobilität, öffentliche Räume aufgezeigt, die alle mit dem weitgehenden Ausschluß von Frauen aus der von Männern besetzten Öffentlichkeit zusammenhängen.

[35] Ihre Befähigung, trotzdem politisch aktiv zu werden, ziehen Frauen aus räumlicher Nähe, den Spezifika der Gecekonduviertel, deren Gassen - wenn auch eingeschränkt nutzbare öffentliche Frauenräume bilden, und v.a. aus ihren informellen Netzwerken, also räumlichen und sozialen Ressourcen (Kaplan 1982 : 547 ; Rabrenovic 1994 : 89, 94 ; Pardo 1990). 
Citation : Wedel, Heidi (2004) 'Alltagsleben und politische Partizipation - Gecekondu-Viertel als gesellschaftlicher Ort', European Journal of Turkish Studies, Thematic Issue No1 - Gecekondu, URL : http://www.ejts.org/document140.html

To quote a passage, use paragraph (§).

\section{Räumliche Ressourcen der Partizipation}

[36] Da das Verlassen des Viertels für Gecekondufrauen keine Selbstverständlichkeit ist, bilden die räumlichen Gegebenheiten ihres Stadtteils einen bestimmenden Faktor für ihre politische Partizipation. Wie strukturiert der materielle Raum in Gecekonduvierteln die soziale Sphäre und ihre Möglichkeiten der politischen Partizipation?

[37] Gecekonduviertel sind relativ geschlossene soziale Orte, in die selten Außenstehende kommen. Unter dem Schutz dieser "dorfähnlichen Familialität" (Joseph 1978 : 541 f.) kann in den Gassen gerade auch für die Frauen eine gewisse, wenn auch klar für Fremde abgegrenzte öffentliche Sphäre entstehen. In Ausdehnung ihres Privatraumes verrichten Frauen hier ihre Arbeit, beobachten, treffen und helfen sich dabei, tauschen Informationen aus und schaffen und pflegen ihre informellen Netzwerke. Trotzdem kann ich der Aussage Kümbetoğlus (1996: 541 f.), daß Gärten und Straßen in Gecekonduvierteln eine ähnliche gesellschaftliche Funktion für Frauen wie die Teehäuser für Männer hätten, nur bedingt zustimmen. Hier kann zwar politische Aktivität im kleinen informellen Kreis stattfinden oder entstehen, nachdem Probleme diskutiert und informell Entscheidungen gefällt worden sind. Es kommen jedoch nur die Frauen der unmittelbaren Nachbarschaft zusammen. Ein Kontakt zu Bewohnerinnen anderer Quartiere und Angehörigen anderer hemşehri-Gruppen findet in den Gassen nicht statt, könnte aber durch einen Wochenmarkt erleichtert werden. Allerdings sind derart offene Räume für längere Versammlungen und Treffen mit Vertreterlnnen politischer Institutionen, bei denen auch die Bewohnerinnen zu Wort kämen, nicht geeignet. Die Gassen werden aber von den politischen Parteien zu Propagandazwecken genutzt, indem mit Lautsprechern sowie Symbolen der Partei und Bildern der Kandidatinnen ausgestattete Autokorsos durch die Viertel geführt werden.

[38] Geschlossene öffentliche Räume stehen den Frauen in Gecekonduvierteln nicht oder nur sehr eingeschränkt zur Verfügung. Deshalb fehlt denjenigen Frauen, die Interesse am Aufbau breiterer sozialer und politischer Kreise haben, die über ihre Verwandtschaft und direkte Nachbarschaft hinausgehen, und die diesbezüglich keinen sozialen und zeitlichen Einschränkungen unterliegen, in der Regel die Möglichkeit, sich zu größeren Versammlungen mit anderen Frauen oder Männern zu treffen. Wie stark dies die Möglichkeiten der Frauen zur politischen Partizipation einschränkt, wird besonders in der Wahlkampfzeit deutlich, wenn die Parteien ihre Veranstaltungen 
Citation : Wedel, Heidi (2004) 'Alltagsleben und politische Partizipation - Gecekondu-Viertel als gesellschaftlicher Ort', European Journal of Turkish Studies, Thematic Issue No1 - Gecekondu, URL : http://www.ejts.org/document140.html

To quote a passage, use paragraph (§).

in den Teehäusern abhalten, die rein männliche Räume sind, zu denen Frauen keinen Zugang haben (Wedel 1999 : 193-203).

[39] Um diesen Ausschluß von Informationen und der Gelegenheit, eigene Forderungen vorzutragen, zumindest teilweise auszugleichen, werden Hochzeitssalons oder Kindergärten für Frauenversammlungen angemietet, wozu eine finanzielle Grundlage bzw. gute Kontakte nötig sind. Aber auch solche Einrichtungen sind in A nicht vorhanden. Dort wurde nach einer großen kollektiven Aktion der Bewohnerinnen vor dem Bezirksrathaus den Frauen, die den Protest weiterentwickeln wollten, vorübergehend ein Teehaus für Versammlungen zur Verfügung gestellt. Dieses Angebot wurde jedoch nach kurzer Zeit von dem Inhaber, einem ANAP-Mitglied, mit Verweis auf seinen rückläufigen Verdienst zurückgenommen - er sagte, die Frauen würden nichts trinken. Weil die Frauen dem weder politische noch finanzielle Macht entgegensetzen konnten, wichen sie in den einzigen öffentlichen Raum im Viertel aus, der ihnen offen stand, nämlich den Korankursraum neben der Moschee. Dies stellte widerum für die Alevitinnen ein abschreckendes Moment dar (Wedel 1999 : 181-186).

[40] Bei fehlenden Alternativen werden Privaträume vorübergehend zu öffentlichen Zwecken umfunktioniert, ohne aber den privaten Charakter ganz zu verlieren: so führen z.B. die Parteien, v.a. ihre Frauenkommissionen, Hausbesuche und -versammlungen durch. Weil die Gecekonduhäuser sehr klein sind und die Einladungen zu diesen Versammlungen privat erfolgen, können auf diese Weise mit hohem Zeitaufwand nur sehr wenig Frauen erreicht werden. Eine Erweiterung der sozialen Kontakte der Frauen, z.B. zu Frauen aus anderen ethnischen oder konfessionellen Gruppen, kann nicht stattfinden. Außerdem sind die Besuche für die Gastgeberinnen mit erhöhter Arbeits- und Kostenbelastung verbunden, weil sie die Wohnung vorher und nachher reinigen müssen und ihre Gäste mit Tee und Gebäck versorgen. Während politische Versammlungen im Teehaus für Männer einen gewissen Freizeitcharakter haben, verstärken Hausversammlungen die häuslichen Pflichten der Frauen. Die Alevitinnen, denen diese Einschränkungen bewußt wurden, forderten im Kommunalwahlkampf öffentliche Räume für Frauen, z.B. Frauencafes oder-werkstätten. 
Citation : Wedel, Heidi (2004) 'Alltagsleben und politische Partizipation - Gecekondu-Viertel als gesellschaftlicher Ort', European Journal of Turkish Studies, Thematic Issue No1 - Gecekondu, URL : http://www.ejts.org/document140.html

To quote a passage, use paragraph (§).

\section{Informelle Netzwerke als soziale Ressource politischer Partizipation}

[41] Angesichts des Fehlens vieler individueller sowie räumlicher Ressourcen gewinnen soziale Ressourcen politischer Partizipation eine entscheidende Bedeutung. Informelle Netzwerke helfen den Gecekondufrauen, politische Informationen zu sammeln, Kontakte aufzubauen und politisch aktiv zu werden. Netzwerke unterstützen die Migrantinnen bei der Integration in die Stadt: bei der Ansiedlung (Hinweise auf Grundstücke, gemeinsamer Gecekondubau, Hilfe bei der Finanzierung, Verteidigung gegen Abriß), bei der ersten, oft illegalen Anlage von Infrastruktur, bei der sozialen Integration ins Quartier und bei der Arbeitssuche sowie in allen späteren Bedarfssituationen. Voraussetzung für die Beteiligung an diesem Netz gegenseitiger Unterstützung ist die Bereitschaft, bei Bedarf selbst zu helfen bzw. der "sozialen Pflicht" nachzukommen ${ }^{6}$. Diese Netzwerke werden täglich neu bestätigt und bekräftigt, wenn Frauen sich tagsüber unangemeldet aufsuchen, wenn Männer sich im Teehaus austauschen und wenn Familien sich abends besuchen.

[42] Ein weiterer "Preis" für die Teilnahme an einem Netzwerk ist ein weitgehender Verzicht auf Intimsphäre in der Familie, da das Haus - im wörtlichen und übertragenen Sinne - immer offen gehalten wird. Für Frauen bedeutet das eine ständige Kontrolle ihrer hausfraulichen Fähigkeiten und Sorgfalt einerseits sowie die Sozialkontrolle ihres Verhaltens außerhalb des Hauses andererseits. Da die nachbarschaftlichen sozialen Netzwerke in Gecekonduvierteln weitgehend mit konfessionell und ethnisch homogenen hemşehri-Gruppen zusammenfallen, werden auf diese Weise soziale Muster der Herkunftsregion inklusive der damit verbundenen traditionellen Autoritätsbeziehungen zwischen den Geschlechtern und Generationen in der Stadt weitergeführt (Ayata 1989 : 111 f.). Sie werden aber allmählich aufgeweicht und verändert.

[43] So unterliegen auch die sozialen Beziehungen und Verhaltensweisen in den Vierteln einem Wandel, der nach Auseinandersetzung und Diskussion schließlich von der community mitgetragen wird. Z.B. sehen Mütter mittlerweile auch für Mädchen eine gute Schulbildung, Berufstätigkeit und ökonomische Unabhängigkeit vom zukünftigen Ehemann sowie die freie Wahl des Ehepartners als erstrebenswert an. Wie selbst Anliegen der feministischen Frauenbewegung wie die Kritik an Gewalt in der Ehe in den informellen Netzwerken aufgegriffen und in der Praxis

6 Sowohl Ayata (1989 : 108) als auch Hoodfar (1988 : 351 f.) streichen heraus, daß kleinere Unterstützungen nicht mit dem hierarchisch konnotierten Begriff der "Hilfe" bezeichnet werden. Dies ist eine zusätzliche Erklärung dafür, warum in quantitativen Studien empfangene Hilfe sehr gering bewertet wird. Zu hemşehrilik als Mobilitätskanal im Wohnungs- und Arbeitssektor siehe Erder (1996 : 261-272). 
Citation : Wedel, Heidi (2004) 'Alltagsleben und politische Partizipation - Gecekondu-Viertel als gesellschaftlicher Ort', European Journal of Turkish Studies, Thematic Issue No1 - Gecekondu, URL : http://www.ejts.org/document140.html

To quote a passage, use paragraph (§).

umgesetzt werden, zeigte sich bei einem Nachbarschaftstreffen (gün) von ca. 15 alevitisch kurdischen Frauen in A :

[44] Bei dem gün wurde die Diskussion zu Gewalt in der Ehe nicht wie ich erwartet hätte - von der studierten, frauenbewegten Nalan, sondern von der Analphabetin Berivan ein - und angeleitet. Sie berichtete zunächst, wie das Kind einer der anwesenden Frauen sich zu ihr geflüchtet und erzählt habe, daß der Vater die Mutter schlage. Nachdem der Wahrheitsgehalt bestätigt wurde, entspann sich eine lebhafte Diskussion über die Illegitimität dieser Gewalt und darüber, wie mit den mißhandelnden Männern umzugehen sei. Berivan führte die Frauen äußerst beharrlich, geschickt und behutsam dahin, nicht Rache zu üben und Gewalt mit der kollektiven physischen Gegengewalt der Frauen zu beantworten, sondern bewußt offene, friedliche Strukturen des Dialogs aufzubauen. Dies setzte sie prompt in die Praxis um, als unerwartet einer der mißhandelnden Ehemänner den Raum betrat. Vor der gesamten Frauenversammlung konfrontierte sie inn mit dem Wissen der Frauengruppe um sein Verhalten, forderte inn zur Stellungnahme auf, um inm dann zu sagen, daß er ungerecht handele und in Zukunft diesbezüglich von den Frauen beobachtet werde. Auf meine spätere Nachfrage, ob daraufhin die betroffene Frau nicht noch mehr geschlagen werde, entgegnete sie, daß die Männer jetzt Angst hätten, weil sie wüßten, daß die Frauen zusammenhalten. Am meisten fürchteten die Männer Öffentlichkeit, deshalb würde auch im Fernsehen oft mit Namensnennung der Täter über Mißhandlung berichtet.

[45] So hat Berivan aus dem Fernsehen gewonnene Erfahrungen über die Öffentlichmachung privater Gewalt erfolgreich auf die Situation in ihrem lokalen sozialen Kontext übertragen, wo informelle Netzwerke eine Öffentlichkeit konstituieren. Letztere können also durchaus zu einem sozialen Wandel in Richtung der Stärkung von Frauen beitragen.

[46] Daneben verdeutlicht dieses Beispiel, wie soziale Netzwerke als Ressource politischer Partizipation von Frauen fungieren können: Berivan hat weder formale Bildung, noch Berufs- und Organisationserfahrung, noch eigenes Geld; ihre räumliche Mobiliät hat sie erst allmählich ausgeweitet. Aber sie hat Selbstvertrauen, das auf ihrer vorbildlichen Haushaltsführung, ihren handwerklichen Fähigkeiten und ihrem großen Beitrag zum Bau und Erhalt des Gecekondus beruht, und hat teil an und fördert selbst das hohe Ansehen, das ihr Mann als Lehrer im Viertel genießt. V.a. aber hat sie ein breites soziales Netzwerk aufgebaut, das über die direkte Nachbarschaft und die eigene hemşehri-Gruppe hinausgeht und auch einige ausgewählte sunnitisch türkische Nachbarinnen, Verwandte in anderen Bezirken der Stadt sowie die politischen Kreise ihres Sohnes 
Citation : Wedel, Heidi (2004) 'Alltagsleben und politische Partizipation - Gecekondu-Viertel als gesellschaftlicher Ort', European Journal of Turkish Studies, Thematic Issue No1 - Gecekondu, URL : http://www.ejts.org/document140.html

To quote a passage, use paragraph (§).

einschließt. Täglich investiert sie mit Unterstützungsleistungen, Ratschlägen, Bewirtungen etc. in das Netzwerk. Umgekehrt erhält sie aus dem Netzwerk nicht nur die nötige Unterstützung bei Tätigkeiten, für die Lese- und Schreibkenntnisse erforderlich sind, sondern soziale Erfahrungen, eine große Menge an Informationen und weiteren Kontakten sowie eigenes Ansehen im Viertel. Dadurch hat sie den informellen Status einer lokalen Wortführerin erhalten. Dieser informelle Status kommt auch beim Kontakt mit formellen politischen Organisationen zum Tragen, was sich z.B. darin äußert, daß die Versammlung mit der SHP-Frauenkommission in ihrer Wohnung stattfindet.

[47] Meine Studie zeigt, daß informelle Netzwerke in türkischen Gecekonduvierteln auf folgenden Ebenen einen Beitrag zur politischen Partizipation leisten:

- [48] Zunächst läßt sich eine indirekte Wirkung feststellen, indem Netzwerke den Teilnehmerinnen Ressourcen politischer Partizipation verschaffen, die ihnen auf formellem Wege nicht zugänglich wären: Status und Selbstvertrauen durch eine aktive Rolle im Netzwerk, finanzielle Ressourcen, Einkommen durch die Vermittlung einer Stelle oder Heimarbeitstätigkeit, Zeit durch Entlastung von Haushaltstätigkeiten und Kinderbetreuung, wenn eine Frau soziale, bürokratische oder politische Kontakte wahrnimmt, politische Bildung durch die Vermittlung von Kontakten zu Vertreterinnen von sozialen Bewegungen und Teilnahme an deren Veranstaltungen. Gecekondufrauen unterstützen wiederum die Bildung von jüngeren Verwandten, indem sie sie im eigenen Haushalt aufnehmen, damit sie in der Großstadt studieren können.

- [49] Informelle Netzwerke fungieren insbesondere als Pool politischer Informationen, indem das geringe Wissen, das einzelnen Frauen aufgrund mangelnder formaler Bildung oder eingeschränkter räumlicher Mobilität und sozialer Kontakte zugänglich ist, zusammengetragen und miteinander geteilt wird. So erhalten die Frauen einen Fundus an Wissen über die neueren politischen Entwicklungen, über die Parteien und ihre Kandidatinnen und deren Positionen, die Aktivitäten und Ziele von politischen Vereinigungen, die Pläne der Bezirkskommune, Zuständigkeitsverteilungen zwischen den Ämtern und Wege, wie diese erreicht werden können, sowie über die Termine von politischen Veranstaltungen.

- [50] Bei informellen Treffen werden zudem kollektive Auffassungen gebildet : Frauen, die sich gut kennen und vertrauen, diskutieren untereinander - bei den alevitischen Kurdinnen auch mit den Männern - über die Vor- und Nachteile einzelner Parteien, die Wirkung eines Wahlboykotts, die Berechtigung der Preise für städtische Dienstleistungen etc. Meine Beobachtungen widerlegen die 
Citation : Wedel, Heidi (2004) 'Alltagsleben und politische Partizipation - Gecekondu-Viertel als gesellschaftlicher Ort', European Journal of Turkish Studies, Thematic Issue $N^{\circ} 1$ - Gecekondu, URL http://www.ejts.org/document140.html

To quote a passage, use paragraph (§).

von Erder getroffene Unterscheidung zwischen informellen Treffen von Familien im häuslichen Bereich, bei denen nur über alltägliche und konkrete Probleme gesprochen werde, einerseits und von Männern im Teehaus, wo über Probleme des Stadtviertels und der eigenen hemşehri-Gruppe diskutiert werde, andererseits (Erder 1996 : 247 f.)

- [51] Informelle Treffen stellen eine Gelegenheit für Frauen dar, Fähigkeiten wie das Sprechen vor einem größeren Kreis, politisches Argumentieren, die Leitung einer Diskussion oder das Schlichten von Konflikten zu erlernen, also civic skills zu erwerben.

- [52] Da Frauen weitgehend aus Entscheidungsmechanismen und den direkten Kontakten mit politischen Funktionsträgern ausgeschlossen sind, versuchen sie, wenigstens indirekt über ihre männlichen Verwandten ihr spezielles Problemwissen, ihre Forderungen und Positionen in die politische Diskussion der Männer einzubringen.

- [53] Bei ihren Treffen beschließen die Frauen auch, in Form kollektiver Selbsthilfe, kollektiver Aktion oder gemeinsamen Vorsprechens bei politischen Stellen selbst aktiv zu werden. Auch beim Vorsprechen spielen Netzwerke eine Rolle: Z.B. gehen eher die Frauen zum Muhtar, die in seiner direkten Nachbarschaft wohnen oder zur selben hemşehri-Gruppe gehören. Im Rathaus wird nicht unbedingt der zuständige, sondern eher der ihnen bekannte Beamte oder Funktionsträger aufgesucht, vorzugsweise direkt der Bürgermeister, denn dieser steht in der Wahrnehmung der Migrantinnen in einem reziproken, klientelistischen Verhältnis zu seinen Wählerinnen: Im Tausch für deren Wählerstimme ist er sozial verpflichtet, sich um ihre kollektiven und individuellen Anliegen zu kümmern.

- [54] Die Kontakte, die Frauen meist über ihre männlichen Verwandten zu Vertreterinnen politischer Initiativen, Organisationen und Institutionen geknüpft haben, werden auch anderen Netzwerkmitgliedern zugänglich gemacht. Bei Behördengängen werden Nachbarinnen bei den Kontaktpersonen persöllich bekannt gemacht oder auf diese hingewiesen. Wird eine Frau von Vertreterlnnen einer Partei oder politischen Initiative zu Hause besucht, ruft sie meist ihr besonders vertraute Nachbarinnen dazu, ein Schneeballsystem, das sowohl von den Gecekondufrauen als auch von den politischen Organisationen gern genutzt wird.

- [55] Schließlich bietet das gemeinsame Vorgehen den Frauen einen gewissen Schutz vor negativen Reaktionen ihrer Männer. Beim Beispiel der Öffentlichmachung von privater Gewalt sollte den betreffenden Männern das Gefühl gegeben werden, daß es für sie peinlich wäre, ihr Verhalten 
Citation : Wedel, Heidi (2004) 'Alltagsleben und politische Partizipation - Gecekondu-Viertel als gesellschaftlicher Ort', European Journal of Turkish Studies, Thematic Issue $N^{\circ} 1$ - Gecekondu, URL http://www.ejts.org/document140.html

To quote a passage, use paragraph (§).

fortzusetzen, und daß sie sich dann in der Gemeinschaft isolieren könnten. In anderen Situationen wird die Grenzüberschreitung von Frauen durch Eindringen in einen bislang männlich besetzten öffentlichen Raum dadurch nicht zum Ehrverlust für einzelne Ehemänner, daß auch die Ehefrauen inrer Nachbarn beteiligt sind. Indem Frauen eines Netzwerkes gemeinsam einen sozialen oder politischen Wandel einleiten, wird es den Männern erleichtert, mitzuziehen, ohne ihr Gesicht gegenüber den anderen betroffenen Männern zu verlieren.

[56] Informelle Netzwerke können also auf vielfältige Weise die lokale Politikpartizipation von Frauen fördern und werden zu diesem Zwecke auch von den politisch interessierten Frauen im Viertel eingesetzt. Aufgrund der Quartierbildung in Gecekonduvierteln sind jedoch die sozialen Netzwerke von Nachbarinnen weitgehend identisch mit hemşehri-Beziehungen.

[57] Zwischen sunnitischen Türkinnen und alevitischen Kurdinnen herrscht auch nach Jahrzehnten des Wohnens im selben Stadtteil eine Atmosphäre gegenseitiger Vorurteile und Mißachtung. Es gibt zwar gewisse soziale und ökonomische Kontakte, jedoch kaum enge Freundschaften. Selbst die Frauen der verschiedenen sunnitisch türkischen hemşehri-Quartiere kennen sich kaum untereinander. Nur dort, wo die Nachbarschaft heterogener ist, haben sie auch Kontakte zu Frauen aus anderen Provinzen, jedoch ebenfalls vorzugsweise innerhalb ihrer eigenen ethnischen und religiösen Gruppe. Die Einschränkung der sozialen Kontakte läßt darauf schließen, daß es eine kollektive Identität nur innerhalb der ethnisch, religiös und meist auch regional homogenen Gruppen im Viertel gibt. Insofern unterscheidet sich die Situation in türkischen Gecekonduvierteln von dem Beiruter Wohnhausviertel, das Suad Joseph untersuchte und wo Angehörige verschiedener ethnischer und religiöser Gruppen in derselben Gasse oder im selben Wohnhaus lebten und diese räumliche Nähe die Überwindung ethnischer Grenzen in heterogenen Frauennetzwerken ermöglichte (Joseph $1978: 545 ; 1983: 7)$. Ethnische Grenzziehung innerhalb von Gecekonduvierteln wird noch dadurch verstärkt, daß sich in der subjektiven Wahrnehmung die soziale Differenzierung mit ethnischen Unterschieden überschneidet und daß Angehörige von ethnisch-religiösen Minderheiten von staatlicher politischer Repression und Übergriffen von islamistischen oder nationalistischen Gruppen bedroht sind. Diese Faktoren zusammen beeinträchtigen die Herausbildung einer auf den Stadtteil bezogenen kollektiven Identität und die gemeinsame Durchführung kollektiver Aktionen. Territoriale kollektive Identität bezieht sich hier auf die relativ kleinen Einheiten der Quartiere. Nicht-territoriale Identitäten, v.a. Ethnizität und 
Citation : Wedel, Heidi (2004) 'Alltagsleben und politische Partizipation - Gecekondu-Viertel als gesellschaftlicher Ort', European Journal of Turkish Studies, Thematic Issue $N^{\circ} 1$ - Gecekondu, URL http://www.ejts.org/document140.html

To quote a passage, use paragraph (§).

Konfessionalismus, werden sowohl von den Bewohnerinnen als auch von politischen Organisationen bei der Konkurrenz um knappe Ressourcen (städtische Dienstleistungen oder Schutz bedrohter ethnischer oder religiöser Minderheiten) für politische Mobilisierung benutzt und spalten eine auf den gesamten Stadtteil bezogene territoriale Identität.

\section{Informelle politische Partizipation: kollektive Aktionen}

[58] Meine Untersuchung zeigt, daß Menschen, die im informellen Sektor wohnen und arbeiten und denen vor allem informelle Ressourcen zur Verfügung stehen, auch eher informell politisch aktiv werden. Kollektive Aktionen zu Infrastrukturproblemen werden überwiegend von Frauen und ihren informellen Netzwerken getragen und mit informellen, oft unkonventionellen, teils illegalen Methoden durchgeführt. Die Frauen blockieren eine Straße oder einen Bagger, damit auch ihre Gasse betoniert wird. Sie „beschlagnahmen“ Kanalisationsrohre oder Strommasten und -kabel, damit auch ihre Gasse angeschlossen wird. Sie mobilisieren informelle Netzwerke, um bei öffentlichen Institutionen kollektiv vorzusprechen. Die für informelle Netzwerke charakteristische Reziprozität wird über klientelistische Beziehungen auch in die formelle Politik ausgedehnt. Diese Partizipationsformen tragen zur Überwindung der künstlichen Spaltung in Öffentlichkeit und Privatheit bei (Blondet 1990, Rabrenovic 1994). Allerdings reagieren Männer auf diesen Wandel oft mit ideologischer (insbesondere bei Islamisten auch faktischer) Ausschlußprogrammatik, indem sie die politischen Aktivitäten der Frauen in den privaten Bereich zurückdefinieren. Weil den Frauen entsprechende Ressourcen fehlen, kommt es nach den Aktionen nicht zu einer Verstetigung oder Organisierung, wohl aber zu mittel- und langfristige Wirkungen. Denn Mißerfolge machen den Frauen ihre Klassen- und Geschlechterdiskriminierung sowie die Grenzen ihres Einflusses innerhalb repräsentativer Demokratieformen bewußt. Sie lernen dabei auch die Zuständigkeiten und Budgetbeschränkungen verschiedener Institutionen kennen. Erfolg kann ihr Selbstvertrauen stärken und ihre Identifikation mit dem Stadtteil erhöhen: „Wir Frauen haben die Probleme gelöst, Infrastruktur in den Stadtteil gebracht". Frauen entdecken sich über die lokale Aktion als politische Subjekte.

[59] Da zu diesen Aktionen innerhalb von einzelnen informellen Netzwerken mobilisiert wird und sie nicht von einem größeren, auf den gesamten Stadtteil bezogenen Zusammenhang getragen werden, werden über sie auch nur die Interessen der Bewohnerinnen einzelner Gassen oder 
Citation : Wedel, Heidi (2004) 'Alltagsleben und politische Partizipation - Gecekondu-Viertel als gesellschaftlicher Ort', European Journal of Turkish Studies, Thematic Issue $N^{\circ} 1$ - Gecekondu, URL http://www.ejts.org/document140.html

To quote a passage, use paragraph (§).

Quartiere verfolgt. Nur bei der Annahme einer Abrißgefahr für den gesamten Stadtteil wurde in meinem Untersuchungsgebiet eine ethnische Grenzziehungen überschreitende gemeinsame kollektive Aktion durchgeführt, die jedoch letztlich an unterschiedlichen ökonomischen und parteipolitischen Interessen auseinanderbrach und nur bedingt zu einer Stärkung der auf den gemeinsamen Wohnraum gestützten territorialen kollektiven Identität führte. Objektiver Bedarf in Verbindung mit subjektiver Deprivation führt zwar zu kollektiven Aktionen, mündet jedoch aufgrund der sozialen Heterogenität nicht in eine formalisiertere, stetige Organisierung z.B. in Form einer Stadtteilorganisation. Parteienkonkurrenz kann einen Anreiz zu kollektivem Engagement für städtische Dienstleistungen bilden, insbesondere wenn die Nutzung klientelistischer Netzwerke Erfolgschancen eröffnet. Sie beeinträchtigt jedoch bei starker und divergierender Partei- (oder BlockJbindung der Bewohnerinnen mittelfristige Zusammenschlüsse der Bewohnerinnen und somit die Entstehung von städtischen sozialen Bewegungen.

[60] Der gemeinsame Wohnraum bildet offensichtlich keine ausreichende kollektive Grundlage. Politische Differenzen in Verbindung mit ethnischen und konfessionalistischen Grenzziehungen werden auch bei Bemühung um kollektive Zusammenhänge auf Basis des Geschlechts nicht überwunden. In meinem Fallbeispiel galt dies selbst für die beteiligten Frauen, die sich explizit von der von Männern betriebenen Machtpolitik und ihren Partei- und Flügelstreitigkeiten distanzierten, um sich für das Gemeinwohl des Stadtteils einzusetzen. So erreichten auch die Frauenversammlungen nach dem mehrtätigen Sitzstreik vor dem Rathaus, die erstmals einen interethnischen Zusammenhang zwischen den Bewohnerinnen des Stadtteils herstellten, keine Kontinuität und stärkere Organisierung. Erschwerend für die Gemeinschaftsbildung kam hinzu, daß gemeinsame geschlechtsspezifische Bedürfnisse bei den Versammlungen nicht thematisiert wurden. Jedoch entstanden durch die aktive und über Mobilisierung hinausgehende führende Beteiligung von Frauen neue weibliche Vorbilder und local leaders, von denen einige später als Kandidatinnen für das Muhtaramt ins Gespräch gebracht wurden.

\section{Formelle politische Partizipation}

[61] Selbst der Kontakt zu formellen politischen Organisationen - Kommunalregierung, Behörden, Parteien und Bewegungsorganisationen - wird weitgehend über informelle Netzwerke vermittelt und mit reziproken Verpflichtungen verknüpft. Er ist stark von klientelistischen 
Citation : Wedel, Heidi (2004) 'Alltagsleben und politische Partizipation - Gecekondu-Viertel als gesellschaftlicher Ort', European Journal of Turkish Studies, Thematic Issue No1 - Gecekondu, URL : http://www.ejts.org/document140.html

To quote a passage, use paragraph (§).

Netzwerkbeziehungen geprägt. "Öffentliche" und "private" Beziehungen sind insbesondere auf der Ebene des Stadtteils eng miteinander verwoben: um besseren Zugang zum Muhtar zu haben und Forderungen über ihn effektiver verfolgen zu können, wird dieser nicht nach Performance, sondern nach Herkunftsgruppe gewählt. Auf der Bezirksebene werden offen städtische Dienstleistungen gegen Wählerstimmen klientelistisch verhandelt. Parteieintritte erfolgen manchmal auch ohne tiefere Kenntnisse des Parteiprogramms, um die informellen Netzwerke in formelle Institutionen hinein auszudehnen und für eigene Zwecke zu nutzen.

[62] Ein erster Zugang von Gecekondufrauen zur Parteiarbeit soll über Frauenkommissionen, die sich auf das besondere Zeitbudget der Frauen und die Einschränkung ihrer räumlichen Mobilität einstellen, erreicht werden, ist jedoch sehr arbeitsintensiv und nur bei hohem Organisationsgrad möglich. Besonders systematische Organisierung wurde von den islamistischen Refah Partei betrieben, die jedoch damals Frauen den Zugang zu formellen politischen Machtpositionen verwehrte. Aber auch in sozialdemokratischen Parteien ist trotz der Einführung von Frauenquoten die Arbeit in Parteigremien, die an berufstätigen Männern ausgerichtet sind, aufgrund der geschlechtsspezifischen Arbeitsteilung in der Familie für verheiratete Frauen mit Kindern faktisch kaum möglich. Die Kandidatur von Frauen wird jedoch von Wählerinnen explizit unterstützt. So wird zunehmend die Forderung nach Frauen in formellen kommunalpolitischen Ämtern diskutiert. Von innen wird enwartet, daß sie das Muhtaramt physisch und im übertragenen Sinne für Frauen zugänglicher machen und neue öffentliche Orte für Frauen wie Frauencafes oder Frauenwerkstätten schaffen. Diese Forderungen werden aber (noch) nicht mit der nötigen Geschlossenheit und Vehemenz gegen die verschiedenen Ausschlußstrategien von Männern durchzusetzen versucht.

\section{Raum, Sphäre und gesellschaftlicher Wandel}

[63] Die räumlichen Gegebenheiten in Gecekonduvierteln verstärken die subjektive Deprivation der Bewohnerinnen. Diese fordern eine verbesserte Befriedigung ihrer Grundbedürfnisse und einen erhöhten Zugang zum politischen Entscheidungsprozeß und wenden sich gegen die Diskriminierung aufgrund von sozioökonomischem Status, Ethnizität und Geschlecht. Dieses Streben nach Emanzipation wird in kollektive Aktionen, die sich auf lokale städtische Probleme, Wohnsicherheit, Infrastrukturen und soziale Einrichtungen beziehen, 
Citation : Wedel, Heidi (2004) 'Alltagsleben und politische Partizipation - Gecekondu-Viertel als gesellschaftlicher Ort', European Journal of Turkish Studies, Thematic Issue No1 - Gecekondu, URL : http://www.ejts.org/document140.html

To quote a passage, use paragraph (§).

transformiert und bei Kontakten zu formellen politischen Organisationen artikuliert. Diese Graswurzelaktivitäten werden jedoch nicht durch Organisierung verstetigt. Dies liegt u.a. daran, daß den stärker von den lokalen Problemen betroffenen Frauen wichtige formelle Ressourcen politischer Partizipation fehlen. Ihre Aktivitäten werden von informellen Netzwerken getragen; die kollektive Identität der Beteiligten bezieht sich außer bei der fundamentalen Bedrohung des ganzen Stadtteils nur auf ethnisch weitgehend homogene Untereinheiten, Quartiere oder einzelne Gassen.

[64] Mißerfolg und Erfolg der Aktionen können die Geschlechterverhältnisse im Viertel verändern. Lokale Aktivitäten können zwar durchaus die Allokation kommunaler Ressourcen beeinflussen und die allmähliche Integration der Stadtteile in die Stadt beschleunigen. Da sie jedoch nicht von dem gesamten Stadtteil und demokratisch konstituierten Organisationen getragen werden und v.a. über klientelistische Verhandlungen zum Erfolg führen, richtet sich diese Ressourcenallokation weniger nach stadtplanerisch durchdachten und das Gemeinwohl berücksichtigenden Kriterien, sondern eher nach dem Verhandlungsgewicht der jeweiligen Anwohnergruppen. Allgemeine, z.B. soziale und ökologische Anliegen geraten dabei aus dem Blickfeld.

[65] Eine Organisierung und Vernetzung auf höherer Ebene wird zudem dadurch erschwert, daß lokale Aktionen überwiegend von Frauen getragen werden, welche schon auf der Ebene des Stadtteils, noch stärker aber auf Bezirks- oder Stadtebene aus politischen Organisations- und Entscheidungsprozessen ausgeschlossen sind. Daß jedoch lokale Aktivitäten in den verschiedenen sozialen Gruppen innerhalb der Gecekonduviertel verbreitet sind, zeigt, welche Potentiale für eine breite städtische soziale Bewegung vorhanden wären, wenn diese Auschlußmechanismen überwunden würden. 
Citation : Wedel, Heidi (2004) 'Alltagsleben und politische Partizipation - Gecekondu-Viertel als gesellschaftlicher Ort', European Journal of Turkish Studies, Thematic Issue N¹ - Gecekondu, URL http://www.ejts.org/document140.html

To quote a passage, use paragraph (§).

\section{References}

Ayata, Sencer (1989) 'Toplumsal Çevre Olarak Gecekondu ve Apartman', Toplum ve Bilim, 46/47, pp. 101-127.

Blondet, Celia (1990) 'Establishing an Identity - Women Settlers in a Poor Lima Neighbourhood', in Jelin, Elizabeth (ed.) Women and Social Change in Latin America, London/New Jersey, United Nations Research Institute for Social Development, pp. 12-46.

Dubetsky, Alan (1977) 'Class and Community in Urban Turkey', in van Nieuwenhuijze, Christoffel A. O. (ed.) Commoners, Climbers and Notables - A Sampler of Studies on Social Ranking in the Middle East, Leiden, Brill, pp. 360-371.

Erder, Sema (1996) İstanbul'a bir Kent Kondu - Ümraniye, Istanbul, İletişim.

Holland-Cunz, Barbara (1992/93) 'Öffentlichkeit und Privatheit - Gegenthesen zu einer klassischen Polarität', Frei Räume - Streitschrift der feministischen Organisation von Planerinnen und Architektinnen, 5, pp. 36-53.

Hoodfar, Homa (1988) Survival Strategies in Low Income Neighbourhoods of Cairo, Ph.D. diss., Dept. of Anthropology, Kent University, Microfiche.

Joseph, Suad (1978) 'Women and the Neighborhood Street in Borj Hammoud, Lebanon', in Beck, Lois ; Keddie, Nikki (eds.) Women in the Muslim World, Cambridge, Harvard University Press, pp. 541-557.

Joseph, Suad (1983) 'Working Class Women's Networks in a Sectarian State: A Political Paradox', American Ethnologist, 10(1), pp. 1-22.

Kaplan, Temma (1982) 'Female Consciousness and Collective Action - The Case of Barcelona, 1910-1918', Signs, 7(3), pp. 545-566.

Karpat, Kemal (1976) The Gecekondu - Rural Migration and Urbanization, Cambridge, Harvard University Press.

Keleş, Ruşen (1983) 100 Soruda Türkiye'de Şehirleşme, Konut ve Gecekondu, Istanbul, Gerçek.

Keleş, Ruşen (1993) Kentleşme Politikası, Ankara, İmge.

Kıray, Mübeccel (1972) 'Gecekondu - Az Gelişmiş Ülkelerde Hızla Topraktan Kopma ve Kentle Bütünleşememe', Ankara Üniversitesi Siyasal Bilimler Fakültesi Dergisi, 27(3), pp. 561-574.

Köksal, Sema (1990) 'Ticarileşen Gecekondu ve Kent Yöneticileri', Marmara Üniversitesi Iktisadi ve İdari Bilimler Fakültesi Dergisi, Prof. Dr. Mübeccel Kıray'a Armağan, 7(1-2), pp. 260-276.

Kümbetoğlu, Belkıs (1996) 'Women in Squatter Settlements and their Living Space', in Komut, Emine M. (ed.) Housing Question of the 'Others', Ankara, Chamber of Architects of Turkey, pp. 86-95.

McCarthy, John D. ; Zald, Mayer N. (1977) 'Resource Mobilization and Social Movements - A Partial Theory', American Journal of Sociology, 82(6), pp. 1212-1241.

Pardo, Mary (1990) 'Mexican American Women Grassroots Community Activists - "Mothers of East Los Angeles"', Frontiers - A Journal of Women Studies, 11(1), pp. 1-7. 
Citation : Wedel, Heidi (2004) 'Alltagsleben und politische Partizipation - Gecekondu-Viertel als gesellschaftlicher Ort', European Journal of Turkish Studies, Thematic Issue No1 - Gecekondu, URL : http://www.ejts.org/document140.html

To quote a passage, use paragraph (§).

Rabrenovic, Gordana (1994) 'Women and Collective Action in Urban Neighborhoods', Urban Affairs Annual Review, 42, pp. 63-76.

Tatıdıdil, Ercan (1989) Kentleşme ve Gecekondu, Izmir, Ege Üniversitesi Edebiyat Fakültesi.

Wedel, Heidi (1999) Lokale Politik und Geschlechterrollen - Stadtmigrantinnen in türkischen Metropolen, Hamburg, Schriften des Deutschen Orient-Instituts. 